\title{
Metabolites of the Ellagitannin Geraniin and Their Antioxidant Activities
}

Author

Affiliation
Hideyuki Ito

Division of Pharmaceutical Sciences, Okayama University Graduate School of Medicine, Dentistry and Pharmaceutical Sciences, Okayama, Japan

\author{
Key words \\ - ellagitannin \\ - geraniin \\ - metabolites \\ urolithin \\ - ORAC \\ - antioxidant activity
}

received Nov. 30, 2010

revised January 13, 2011

accepted January 15, 2011

\section{Bibliography}

Dol http://dx.doi.org/ 10.1055/s-0030-1270749 Published online February 3, 2011

Planta Med 2011; 77 :

1110-1115 @ Georg Thieme

Verlag KG Stuttgart · New York . ISSN 0032-0943

\section{Correspondence}

Assoc. Prof. Hideyuki Ito

Division of

Pharmaceutical Sciences

Okayama University

Graduate School of

Medicine, Dentistry

and Pharmaceutical Sciences

1-1-1 Tsushimanaka

Kita-ku

Okayama 700-8530

Japan

Phone: +81862517937

Fax: +81862517937

hito@cc.okayama-u.ac.jp

\section{Abstract \\ $\nabla$}

Different types of ellagitannins are reported to have various biological activities, such as antioxidant, antiviral, and antitumor activities. However, there are few definitive studies on the absorption and metabolism of ellagitannins. This review compares the absorption and metabolism of ellagitannins, and the antioxidant properties of their metabolites in rats, with those of intact ellagitannins by means of in vitro and in vivo assays. We isolated 7 urinary and intestinal microbial metabolites in rats after the ingestion of geraniin, which is a typical ellagitannin isolated from Geranium thunbergii, an antidiarrheic remedy in Japan. The structures of these metabolites were determined to be dibenzopyran derivatives (1-7), using NMR and mass spectroscopic data. Four major metabo-

\section{Introduction}

$\checkmark$

Tannins are polyphenols that are widespread constituents of vegetables and are classified into 2 groups: hydrolyzable tannins and condensed tannins [1]. Ellagitannins belong to the hydrolyzable tannin group and occur in foods such as raspberries, strawberries, blackberries, pomegranate, almonds, and walnuts [2]. In vitro and in vivo studies of ellagitannins demonstrate various biological activities, including antioxidant [3], antiviral [4], antimutagenic [5], antimicrobial [6,7], and antitumor promotion activities [8,9], suggesting that the consumption of ellagitannins confers health benefits to humans. Nevertheless, the bioavailability of purified ellagitannins after ingestion is not fully understood. Ellagic acid is one of the hydrolysates of ellagitannins; its metabolism has been previously studied by Doyle and Griffiths [10]. The metabolite in urine and feces after oral administration of ellagic acid to rats is characterized as 3,8-dihydroxy-6H-dibenzo[b,d]pyran- lites (1-4) prepared by chemical synthesis were evaluated for their antioxidant activities by using 2,2-diphenyl-1-picrylhydrazyl radical scavenging and oxygen radical absorbance capacity (ORAC) methods. The metabolites exhibited more potent antioxidant activities in the ORAC assay than intact ellagitannins, such as geraniin and corilagin. Furthermore, plasma ORAC scores increased with increases in the plasma concentration of the metabolites after the oral administration of geraniin to rats. These findings suggest that these metabolites may contribute to the health benefits of ellagitannins as antioxidants in the body.

Supporting information available online at http://www.thieme-connect.de/ejournals/toc/ plantamedica

6-one (urolithin A). The absorption and metabolism of punicalagin from pomegranate has recently been reported in rats [11] and humans [12-14]. The metabolism of ellagitannins from several foodstuffs has also been demonstrated in humans [15]. Although the presence of various metabolites in plasma or urine after the ingestion of juice from fruits rich in ellagitannins is suggested, the chemical structures of these metabolites (except for urolithin $\mathrm{A}$ ) have not been clearly characterized.

Geraniin (8) is the main polyphenolic component in Geranuim thunbergii, a medicinal plant used to treat diarrhea in Japan. Several biological studies of geraniin show that it possesses antioxidant, antitumor, and antivirus properties. Geraniin is a typical ellagitannin because it is composed entirely of common acyl units such as galloyl, hexahydroxydiphenoyl (HHDP), and dehydrohexahydroxydiphenoyl (DHHDP) groups. We used geraniin as a purified ellagitannin to study the bioavailability of ellagitannins. Seven metabolites, 


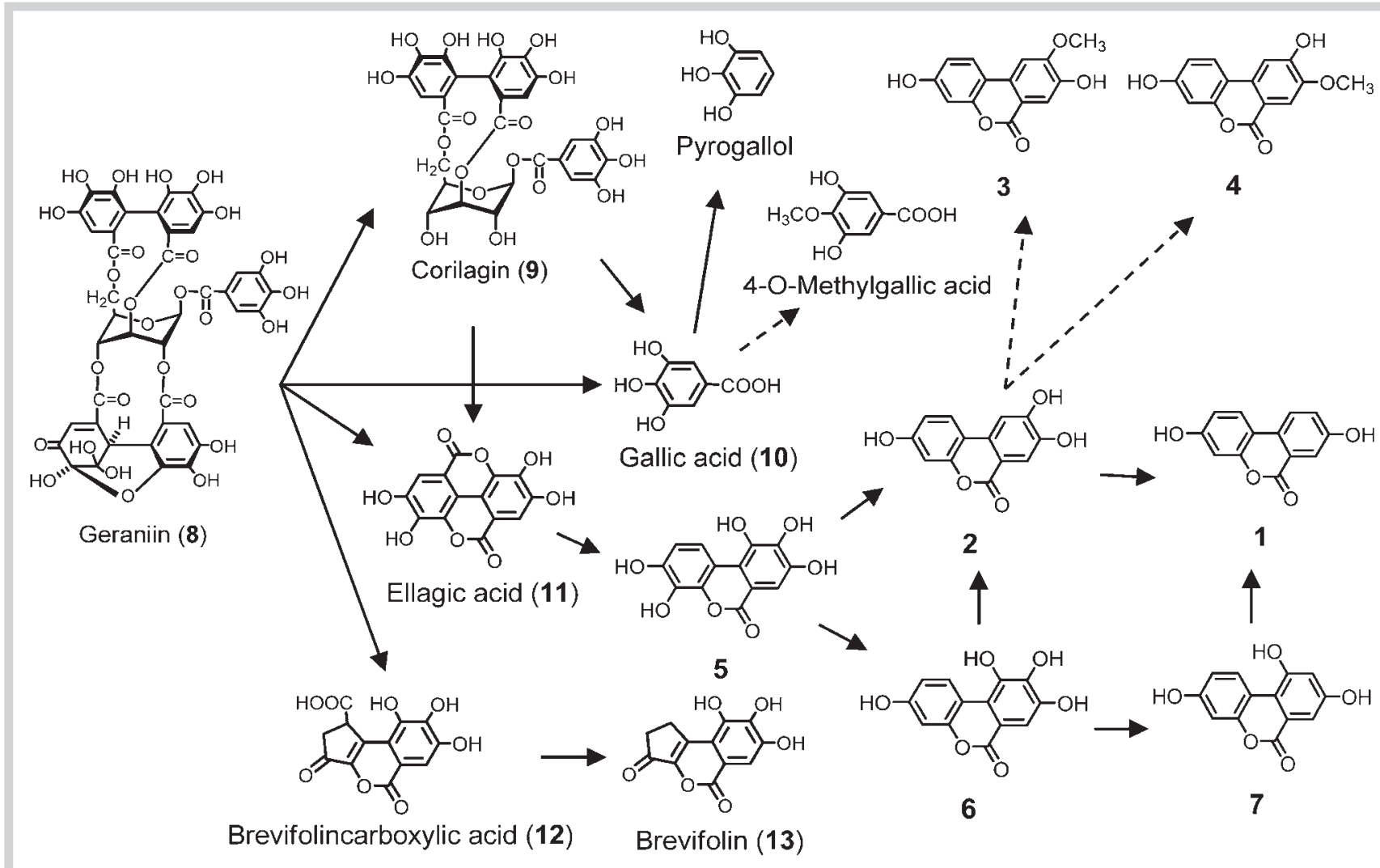

Fig. 1 Proposed metabolic pathway for the formation of metabolites originating from geraniin. Solid and dotted arrows represent possible reactions by intestinal microflora and catechol-O-methyltransferase (COMT), respectively.

including urolithin $\mathrm{A}$, were isolated from rat intestinal microbial suspensions with geraniin and rat urine after oral administration of geraniin. The structures of these metabolites were elucidated using NMR and mass spectral data. We also investigated the absorption of intact ellagitannins and urinary recovery of the metabolites in rats [16]. Furthermore, the antioxidant properties of ellagitannin metabolites were evaluated and compared with those of the intact ellagitannins and related polyphenols by using both in vitro and in vivo methods. In this review, the metabolic fate of ellagitannins determined through isolation and structural elucidation, as well as the association between antioxidant properties and plasma levels of ellagitannin metabolites are discussed briefly.

\section{Isolation and Structural Elucidation of Ellagitannin Metabolites from Rat Biofluids $\nabla$}

The functional constituents of medicinal plants or foods after oral dosing may be primarily affected by intestinal microflora before absorption in the gut. Geraniin (8) was anaerobically incubated in a suspension of rat intestinal microflora. After a 96-h incubation, the suspension was filtered and subjected to column chromatography and/or preparative HPLC to give 5 metabolites: 1, 2, and 57. Urine samples from rats were collected for $48 \mathrm{~h}$ after oral administration of geraniin, incubated with $\beta$-glucuronidase and sulfatase, and separated by repeated column chromatography to afford 4 major metabolites: 1-4.
The isolated metabolites from rat biofluids were identified as follows: 3,8-dihydroxy-6H-dibenzo[b,d]pyran-6-one (1; urolithin A) [17], 3,8,9-trihydroxy-6H-dibenzo[b,d]pyran-6-one (2) [18], 3,4,8,9,10-pentahydroxy-6H-dibenzo[b,d]pyran-6-one (5) [19], 3,8,9,10-tetrahydroxy-6H-dibenzo[b,d]pyran-6-one (6), and 3,8,10-trihydroxy-6H-dibenzo[b,d]pyran-6-one (7) [20] by comparing spectroscopic data with those reported in the literature ( Fig. 1). Both metabolites $\mathbf{3}$ and $\mathbf{4}$ were characterized as dibenzopyran derivatives carrying a methoxy unit. The positions of each methoxy group in metabolites $\mathbf{3}$ and $\mathbf{4}$ were established using nuclear Overhauser enhancement spectroscopy (NOESY) experiments. The structures of $\mathbf{3}$ and $\mathbf{4}$ were determined to be 3,8dihydroxy-9-methoxy-6H-dibenzo[b,d]pyran-6-one and 3,9-dihydroxy-8-methoxy-6H-dibenzo[b,d]pyran-6-one [17], respectively (๑ Fig. 1).

Among the ellagitannin metabolites, metabolite 1 has been previously isolated and characterized from rat urine and feces after oral administration of ellagic acid [10] and was also identified as a metabolite in sheep serum and urine after the consumption of Terminalia oblongata leaves, which contain abundant amounts of ellagitannins [1]. Metabolite 1 has also been characterized as castoreum pigment I from the scent glands of beavers [21], a constituent of renal calculi in sheep [22], and a plant ingredient of Shilajit [23] and Trapa natans [24]. Furthermore, metabolite 1 was recently found to be a urinary metabolite in humans after pomegranate juice supplementation [14]. Metabolites 5 and 7 have been identified as phytochemicals from Tamarix nilotica [19] and as metabolites from the feces of Trogopterus xanthipes [20], 
respectively. Compounds 2-7 were unambiguously characterized as ellagitannin metabolites for the first time in our study.

\section{Rat Intestinal Microbial Transformation of Ellagitannins}

The time-course profiles of the intestinal microbial transformation of geraniin were investigated using reversed-phase HPLC. The proposed metabolic pathway of geraniin is summarized in - Fig. 1. Geraniin (8) begins to be converted to corilagin (9), gallic acid (10), ellagic acid (11), and brevifolincarboxylic acid (12) after $1 \mathrm{~h}$ of anaerobic incubation with rat fecal suspension, indicating that geraniin is first hydrolyzed by microflora. Metabolite $\mathbf{5}$ was produced 6 h after incubation; metabolites 2 and 6 were subsequently detected after a 48-h incubation. Upon further incubation, transformation to metabolites 1 and 7 occurred after $96 \mathrm{~h}$. Similar observations to those of geraniin were made after the reincubation of both corilagin (9) and ellagic acid (11) among the intermediate hydrolysates. Furthermore, brevifolincarboxylic acid (12) was decarboxylated to brevifolin (13) during reincubation with gut microflora. Geraniin was first subjected to ester hydrolysis with a rat fecal suspension, to give corilagin, and gallic, ellagic, and brevifolincarboxylic acids, indicating that geraniin is similarly hydrolyzed under acidic conditions [25]. Among the hydrolysates, the metabolism of ellagic acid derived from HHDP and DHHDP [26,27] groups was expanded to metabolites $\mathbf{1}, \mathbf{2}$, and 7 through metabolite 5 by the fission of 1 of 2 lactone rings and subsequent dehydroxylation.

\section{Urinary Excretions of Intact Ellagitannins and Their Metabolites in Rats after Oral Dosing of Ellagitannins $\nabla$}

The collected urine samples were treated with $\beta$-glucuronidase and sulfatase after oral administration of ellagitannins or their related polyphenols to rats. Urinary excretion of metabolites has been demonstrated by reversed-phase HPLC analysis. The identity of each metabolite is based on their individual retention times, which were compared to respective standards. The microbial metabolites, $\mathbf{1}$ and 2, and metabolites having a methoxy group, metabolites $\mathbf{3}$ and $\mathbf{4}$, were markedly excreted in urine after geraniin consumption and were identified as major metabolites. The cumulative urinary excretion of major metabolites (1-4) after oral administration of geraniin is shown in 0 Fig. 2. All metabolites started to be detected in rat urine $24 \mathrm{~h}$ after geraniin intake; their excretion increased until $72 \mathrm{~h}$. The total excretion of major metabolites in urine over $72 \mathrm{~h}$ reached $12.4 \%$ of the amount administered at $20 \mathrm{mg} /$ head. Among these, large amounts of metabolite $\mathbf{2}$ were recovered in urine at a level of $3.56 \mu \mathrm{mol} / 72 \mathrm{~h}(6.8 \%$ of the oral dose of geraniin). The urinary excretion of all metabolites persisted for more than $48 \mathrm{~h}$ after geraniin administration. This suggests that ellagitannin metabolites formed by microbial hydrolysis and subsequently reduced in the colon are absorbed and eliminated via enterohepatic circulation. These data reinforce the notion that the metabolism of ellagitannins is dependent on intestinal microflora $[11,13]$.

These metabolites were also detected in urine samples after oral administration of either corilagin (9) or ellagic acid (11). After oral dosing of brevifolincarboxylic acid (12), which is a hydrolysate from geraniin, brevifolin (13) due to the decarboxylated derivative of 12 was observed in urine until $12 \mathrm{~h}$. The major metab-

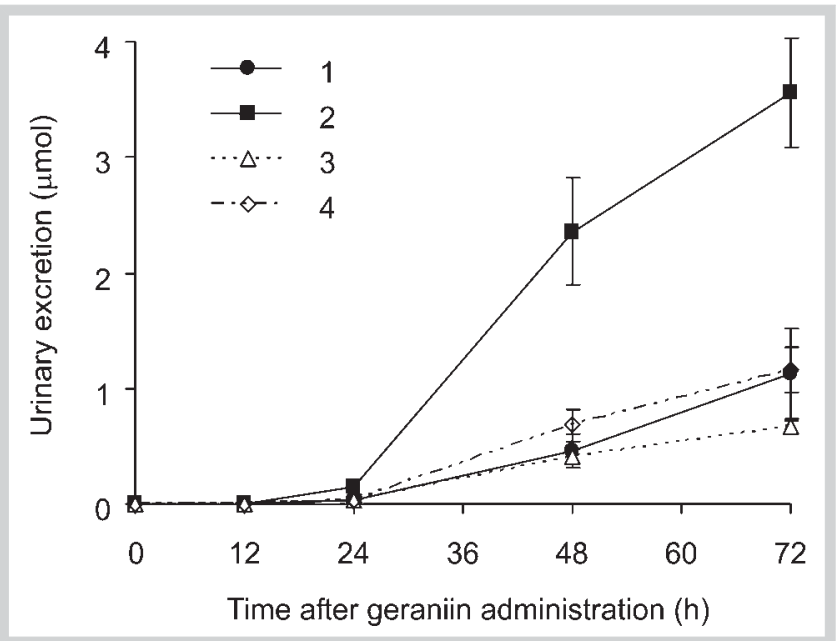

Fig. 2 Cumulative urinary excretion profile of major metabolites in rats after oral administration (50 mg/head) of geraniin. Urine was collected from metabolic cages $0-12,12-24,24-48$, and $48-72 \mathrm{~h}$ after administration. Values are means $(S E M)(n=4)$ represented by vertical bars.

olites were scarcely detected in urine samples that were not treated with $\beta$-glucuronidase and sulfatase after geraniin ingestion. However, several other weakly retained peaks were observed on reversed-phase HPLC analysis, suggesting that all metabolites are almost excreted in urine as conjugate forms.

The transformations to both metabolites $\mathbf{3}$ and $\mathbf{4}$ (identified as urinary metabolites) were not observed in all rat fecal suspensions with ellagitannins. The methylation of polyphenols with a catechol unit by catechol- 0 -methyltransferase (COMT) is well known among flavonoids and catechins [28]. The production of metabolites $\mathbf{3}$ and $\mathbf{4}$ was attributed to the methylation of $\mathbf{2}$ absorbed from the intestines by COMT in the liver or kidneys. Pyrogallol and 4-O-methylgallic acid have been identified as metabolites of gallic acid in both humans [29] and rats [30]. Based on these findings, a metabolic sequence is proposed for the formation of these metabolites that originate from geraniin ( $\bullet$ Fig. 1 ). We further investigated the presence of intact ellagitannins in rat urine after oral administration by using HPLC-ESI-MS/MS. Corilagin was detected in rat urine after consumption of geraniin, suggesting that it is absorbed and excreted in urine after gut microbial elimination of the DHHDP group in geraniin. In addition, corilagin was clearly observed in rat urine after its intake. The presence of condensed tannins, such as proanthocyanidins, in rat plasma after apple polyphenol consumption is reported [31-33]. Although the absorption of hydrolyzable tannins in both animals and humans is not well understood at present, these findings demonstrate that hydrolyzable tannins having molecular weights $>600$ can be absorbed and excreted after oral dosing.

\section{Ellagitannin Metabolites in Rat Serum after Oral Dosing of Geraniin $\nabla$}

Rat serum levels of the ellagitannin metabolites after oral administration of geraniin were analyzed by HPLC-ESI-MS/MS. All of the major metabolites found in rat urine were also detected in rat serum. Among the metabolites, serum metabolite $\mathbf{2}$ contents were highest similarly to the urinary excretion. Serum metabolite 
Table 1 2,2-Diphenyl-1-picrylhydrazyl (DPPH) radical scavenging and superoxide dismutase (SOD)-like activities of ellagitannins and their metabolites.

\begin{tabular}{|c|c|c|c|}
\hline \multirow[t]{3}{*}{ Compounds } & \multirow[t]{3}{*}{ DPPH IC $C_{50}(\mu \mathrm{M})$} & \multicolumn{2}{|l|}{ SOD } \\
\hline & & Xanthine-XOD & PMS-NADH \\
\hline & & $\mathrm{IC}_{50}(\mu \mathrm{M})$ & $\mathrm{IC}_{50}(\mu \mathrm{M})$ \\
\hline 1 & $>100$ & $>100$ & $>100$ \\
\hline 2 & 1.9 & $>100$ & 51 \\
\hline 3 & 11 & $>100$ & $>100$ \\
\hline 4 & 62 & $>100$ & $>100$ \\
\hline Geraniin (8) & 0.6 & 0.8 & 1.3 \\
\hline Corilagin (9) & 0.4 & 0.8 & 0.7 \\
\hline Gallic acid (10) & 1.9 & 13 & 7.8 \\
\hline Ellagic acid (11) & I) 1.7 & $>100$ & 21 \\
\hline Pyrogallol & 2.1 & 3.5 & 4.7 \\
\hline
\end{tabular}

2 and 1 levels reached a maximum after 24 and 48 h, respectively (- Fig. 3). This implies that metabolite $\mathbf{1}$ is dehydroxylated from 2 by gut microflora, which in turn is absorbed into enterohepatic circulation. Since a trace level of these metabolites was observed in rat serum samples without enzymatic treatment with $\beta$-glucuronidase, the ellagitannin metabolites almost existed in rat serum in glucuronide or sulfate forms. Although the metabolites are present in the circulatory system in their conjugated forms, much data exist regarding the biological activity of these conjugates. Quercetin conjugates retain antioxidant activity in plasma [34-36]. Luteolin glucuronide is deconjugated into the aglycone by $\beta$-glucuronidase released from neutrophils after the induction of inflammation [37]. Thus, these findings support the idea that these metabolites are absorbed into the circulatory system and are transported in urine after ellagitannin intake, which may contribute to its health benefits.

\section{Association between Ellagitannin Metabolite Plasma Levels and Antioxidant Activities \\ $\nabla$}

The major metabolites $\mathbf{1 - 4}$ were prepared by condensing resorcinol with bromohydroxybenzoic acid in alkaline solution with a copper catalyst [16]. The resultant products were used to evaluate antioxidant activity. The antioxidant activities of the major metabolites were evaluated by 2,2-diphenyl-1-picrylhydrazyl (DPPH) radical scavenging effects and 2 superoxide dismutase (SOD)-like activities ( Table 1). Superoxide anions were either generated by a xanthine-XOD enzymatic reaction or by a phenazine methosulfate (PMS)-NADH nonenzymatic reaction. In both of the assays for SOD-like activity, none of the major metabolites exhibited activity except for $\mathbf{2}$ in the PMS-NADH assay $\left(\mathrm{IC}_{50}=\right.$ $51 \mu \mathrm{M})$. On the other hand, among the tested metabolites, metabolite 2 had a radical scavenging effect with an $\mathrm{IC}_{50}$ of $1.9 \mu \mathrm{M}$, which is comparable to that of geraniin and its related polyphenols in the DPPH assay. These data suggest that metabolite 2 possesses radical scavenging activity due to the presence of a catechol unit in the molecule.

The oxygen radical absorbance capacity (ORAC) method is a standard recommended by the United States Department of Agriculture for measuring antioxidant capacity and has the advantage of utilizing a biologically relevant radical source $[38,39]$. We measured the antioxidant capacity of ellagitannin metabolites in relation to intact ellagitannins by using the ORAC method (๑ Fig. 4). The intact ellagitannins and their related polyphenols exhibited

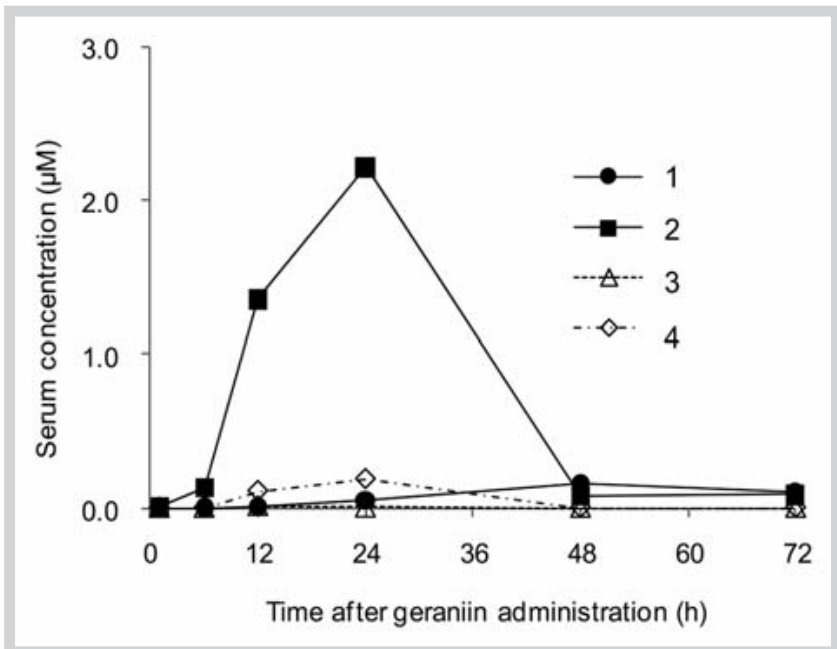

Fig. 3 Serum levels of major metabolites in rats after oral administration $(20 \mathrm{mg} /$ head) of geraniin $(n=2-3)$.

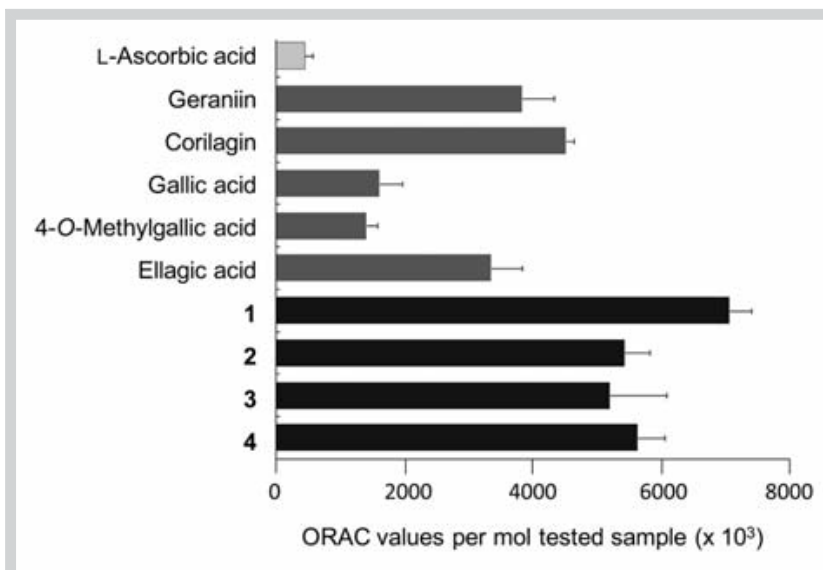

Fig. 4 Oxygen radical absorbance capacity (ORAC) values per mole of intact ellagitannins and their metabolites $(n=3) .{ }^{* *} \mathrm{P}<0.01$; statistically significant differences between each compound were evaluated by 1-way ANOVA.

strong antioxidant activities compared to ascorbic acid. The ORAC scores of all metabolites indicate potent antioxidant properties compared to those of intact ellagitannins. Furthermore, the ORAC potencies of $\mathbf{1}, \mathbf{3}$, and $\mathbf{4}$ without catechol units were significantly higher than those of intact ellagitannins. Metabolite 1 was the most potent among the tested metabolites. This result suggests that the metabolites may function as biological antioxidants after ingestion of ellagitannins.

The antioxidant properties of the ellagitannin metabolites were evaluated using an in vivo study. We investigated the association between metabolite plasma levels and plasma ORAC scores after geraniin consumption in rats. The collected rat plasma samples after geraniin oral administration at $5 \mathrm{mg} /$ head were extracted with methanol containing hydrochloric acid. The plasma levels of the metabolites of the samples were analyzed using HPLC-ESI-MS/MS and ORAC scores were subsequently assayed. In this case, metabolite 1 was the main metabolite detected in rat plasma after a low dose of geraniin. However, metabolite $\mathbf{2}$ was 


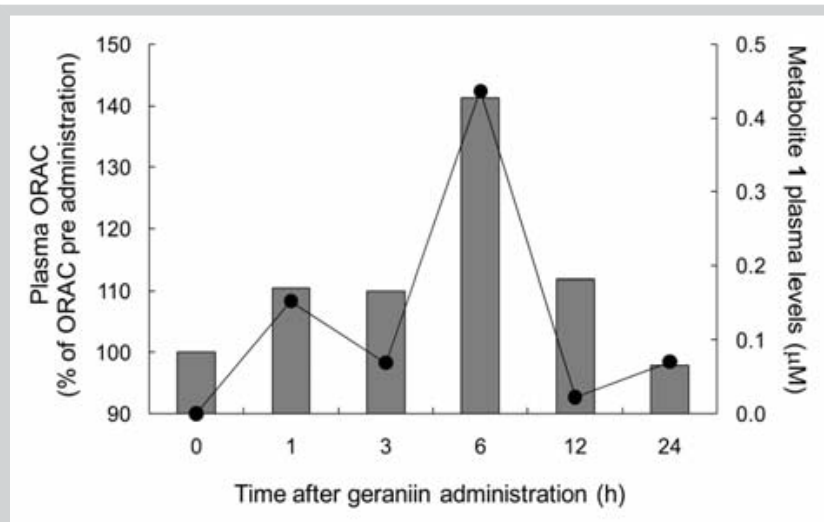

Fig. 5 Plasma levels of 1 and plasma ORAC scores after oral administration $(20 \mathrm{mg} /$ head) of geraniin $(n=3)$. Lines represent metabolite 1 plasma levels and bars represent percentages of plasma ORAC scores before geraniin administration.

mainly found in rat serum after a high dose $(20 \mathrm{mg} / \mathrm{head})$ of geraniin, as mentioned above. The different main metabolites found in rat blood at low and high doses suggest that the gut microbial conversion of $\mathbf{2}$ to $\mathbf{1}$ may be saturated at high doses. The plasma concentration of 1 reached a maximum $6 \mathrm{~h}$ after administration. Plasma ORAC scores simultaneously reached their maximum, indicating a strong association between plasma metabolite $\mathbf{1}$ levels and the plasma ORAC scores ( $\mathbf{O}$ Fig. 5). This result also indicates that ellagitannin metabolite $\mathbf{1}$ possesses antioxidant activity in vivo as well as in vitro. These findings raise the possibility that these metabolites play an important role in biological antioxidants after the oral administration of intact ellagitannins as potent natural antioxidants.

\section{Conclusions}

$\nabla$

Ellagitannins such as geraniin and punicalagin have a hexahydroxydiphenoyl group in their molecules; consequently, ellagic acid, which is readily lactonized from hexahydroxydiphenic acid, is produced upon the hydrolysis of ellagitannins [25]. Metabolic profiles suggest that ellagitannin metabolites are derived from ellagic acid via ellagitannin hydrolysis. These metabolites were recently found in the biofluids of rats [10,11,40-43], mice [44], and humans $[13,15,45-47]$ in in vivo studies on the functionalities of foodstuffs rich in ellagitannins, including pomegranates, nuts, and berries. Most of the reported unidentified ellagitannin metabolites may correspond to metabolites 1-7 and/or their conjugate forms. The ORAC method revealed that the antioxidant properties of major metabolites 1-4 are more potent than those of intact ellagitannins. A close relationship between plasma metabolite 1 levels and plasma ORAC scores was observed after the oral administration of geraniin. Urolithin A (1) was recently reported to possess several biological activities such as inhibition of prostate cancer [44], anti-inflammatory effects [48], as well as systematic health benefits related to the consumption of ellagitannins. Further study of the biological properties of ellagitannin metabolites, including the bioavailability of ellagitannins, is necessary to clarify the active principles of several biological activities of ellagitannins such as their antioxidant and chemopreventive effects.
Most evaluations of the biological activities of natural products are targeted toward plant components. Our findings raise the possibility that metabolites play important roles in biological antioxidants after oral administration of intact ellagitannins. Studying the bioavailability of natural products is important to clarify the bioactive principles of their biological activities.

\section{Supporting information}

Ion chromatograms of corilagin in rat urine samples obtained by HPLC-ESI-MS/MS in the multiple reaction-monitoring mode with negative ionization are available as Supporting Information.

\section{Acknowledgements}

$\nabla$

This study was supported in part by a Grant-in-Aid for Scientific Research (No.17604005) from the Ministry of Education, Culture, Sports, Science, and Technology of Japan and by the Mishima Kaiun Memorial Foundation. The author would like to thank Professors Takuo Okuda, Takashi Yoshida, and Tsutomu Hatano in addition to Drs. Akihiro Tai, Yoshiaki Amakura, and Morio Yoshimura for their helpful advice. The author would also like to thank Mr. Hidekazu Ishimoto and Mr. Ayumu Iguchi for their contribution to this work.

\section{References}

1 Okuda T, Yoshida T, Hatano T. Hydrolyzable tannins and related polyphenols. Fortschr Chem Org Naturs 1995; 66: 1-117

2 Clifford MN, Scalbert A. Ellagitannins, occurrence in food, bioavailability and cancer prevention. J Food Sci Agric 2000; 80: 1118-1125

3 Okuda T, Yoshida T, Hatano T. Ellagitannins as active constituents of medicinal plants. Planta Med 1989; 55: 117-122

4 Nakashima H, Murakami T, Yamamoto N, Sakagami H, Tanuma S, Hatano T, Yoshida T, Okuda T. Inhibition of human immunodeficiency viral replication by tannins and related compounds. Antiviral Res 1992; 18 : 91-103

5 Okuda T, Mori K, Hayatsu H. Inhibitory effect of tannins on direct-acting mutagens. Chem Pharm Bull 1984; 32: 3755-3758

6 Funatogawa K, Hayashi S, Shimomura H, Yoshida T, Hatano T, Ito H, Hirai $Y$. Antibacterial activity of hydrolyzable tannins derived from medicinal plants against Helicobacter pylori. Microbiol Immunol 2004; 48: 251-261

7 Shiota S, Shimizu M, Mizusima T, Ito H, Hatano T, Yoshida T, Tsuchiya T. Restoration of effectiveness of $\beta$-lactams on methicillin-resistant Staphylococcus aureus by tellimagrandin I from rose red. FEMS Microbiol Lett 2000; 185: 135-138

8 Ito H, Miyake M, Nishitani E, Mori K, Hatano T, Okuda T, Konoshima T, Takasaki M, Kozuka M, Mukainaka T, Tokuda H, Nishino H, Yoshida T. Anti-tumor promoting activity of polyphenols from Cowania mexicana and Coleogyne ramosissima. Cancer Lett 1999; 143: 5-13

9 Okabe S, Suganuma M, Imayoshi Y, Taniguchi S, Yoshida T, Fujiki H. New TNF- $\alpha$ releasing inhibitors, geraniin and corilagin, in leaves of Acer nikoense, Megusurino-ki. Biol Pharm Bull 2001; 24: 1145-1148

10 Doyle B, Griffiths LA. The metabolism of ellagic acid in the rat. Xenobiotica 1980; 10: 247-256

11 Cerda B, Llorach R, Ceron JJ, Espin JC, Tomas-Barberan FA. Evaluation of the bioavailability and metabolism in the rat of punicalagin, an antioxidant polyphenol from pomegranate juice. Eur J Nutr 2003; 42: 18-28

12 Seeram NP, Lee R, Heber D. Bioavailability of ellagic acid in human plasma after consumption of ellagitannins from pomegranate (Punica granatum L.) juice. Clin Chim Acta 2004; 348: 63-68

13 Seeram NP, Henning SM, Zhang Y, Suchard M, Li Z, Heber D. Pomegranate juice ellagitannin metabolites are present in human plasma and some persist in urine for up to 48 hours. J Nutr 2006; 136: 2481-2485

14 Cerda B, Espin JC, Parra S, Martinez P, Tomas-Barberan FA. The potent in vitro antioxidant ellagitannins from pomegranate juice are metabolised into bioavailable but poor antioxidant hydroxy-6H-dibenzopyran-6-one derivatives by the colonic microflora of healthy humans. Eur J Nutr 2004; 43: 205-220 
15 Cerda B, Tomas-Barberan FA, Espin JC. Metabolism of antioxidant and chemopreventive ellagitannins from strawberries, raspberries, walnuts, and oak-aged wine in humans: identification of biomarkers and individual variability. J Agric Food Chem 2005; 53: 227-235

16 Ito $H$, Iguchi A, Hatano $T$. Identification of urinary and intestinal bacterial metabolites of ellagitannin geraniin in rats. J Agric Food Chem 2008; 56: 393-400

17 Pandey J, Jha AK, Hajela K. Synthesis and biological activities of some new dibenzopyranones and dibenzopyrans: search for potential oestrogen receptor agonists and antagonists. Bioorg Med Chem 2004; 12: 2239-2249

18 Ghosal S, Lata S, Kumar Y. Free radicals of shilajit humus. Indian J Chem 1995; 34B: 591-595

19 Nawwar MAM, Souleman AMA. 3,4,8,9,10-Pentahydroxy-dibenzo[b, d]pyran-6-one from Tamarix nilotica. Phytochemistry 1984; 23: 2966-2967

20 Jeong SJ, Kim NY, Kim DH, Kang TH, Ahn NH, Miyamoto T, Higuchi R, Kim YC. Hyaluronidase inhibitory active $6 H$-dibenzo[b,d]pyran-6-ones from the feces of Trogopterus xanthipes. Planta Med 2000; 66: 76-77

21 Lederer $E$. Chemistry and biochemistry of some mammalian secretions and excretions. J Chem Soc 1949: 2115-2125

22 Pope GS. Isolation of two benzocoumarins from 'clover stone' a type of renal calculus found in sheep. Biochem J 1964; 93: 474-477

23 Ghosal S, Reddy JP, Lal VK. Shilajit I: chemical constituents. J Pharm Sci 1976; 65: 772-773

24 Shirataki Y, Yoshida S, Toda S. Dibenzo- $\alpha$-pyrons in fruits of Trapa natans. Natural Medicines 2000; 54: 160

25 Okuda T, Yoshida T, Hatano T. Constituents of Geranium thunbergii Sieb. et Zucc. Part 12. Hydrated stereostructure and equilibration of geraniin. J Chem Soc [Perkin I] 1982: 9-14

26 Okuda T, Hatano T, Nitta H, Fujii R. Hydrolysable tannins having enantiomeric dehydrohexahydroxydiphenoyl group: revised structure of terchebin and structure of granatin B. Tetrahedr Lett 1980; 21: 43614364

27 Schmidt OT, Schulz J, Wurmb R. Über natürliche Gerbstoffe, XXXVI. Terchebin. Justus Liebigs Ann Chem 1967; 706: 169-179

28 Manach C, Scalbert A, Morand C, Remesy C, Jimenez L. Polyphenols: food sources and bioavailability. Am J Clin Nutr 2004; 79: 727-747

29 Hodgson JM, Morton LW, Puddey IB, Beilin LJ, Croft KD. Gallic acid metabolites are markers of black tea intake in humans. J Agric Food Chem 2000; 48: 2276-2280

30 Yasuda T, Inaba A, Ohmori M, Endo T, Kubo S, Ohsawa K. Urinary metabolites of gallic acid in rats and their radical-scavenging effects on 1,1diphenyl-2-picrylhydrazyl radical. J Nat Prod 2000; 63: 1444-1446

31 Baba S, Osakabe N, Natsume M, Terao J. Absorption and urinary excretion of procyanidin B2 [epicatechin-( $4 \beta-8)$-epicatechin] in rats. Free Radic Biol Med 2002; 33: 142-148

32 Sano A, Yamakoshi J, Tokutake S, Tobe K, Kubota Y, Kikuchi M. Procyanidin B1 is detected in human serum after intake of proanthocyanidinrich grape seed extract. Biosci Biotechnol Biochem 2003; 67: 11401143

33 Shoji T, Masumoto S, Moriichi N, Akiyama H, Kanda T, Ohtake Y, Goda Y. Apple procyanidin oligomers absorption in rats after oral administration: analysis of procyanidins in plasma using the porter method and high-performance liquid chromatography/tandem mass spectrometry. J Agric Food Chem 2006; 54: 884-892
34 Manach C, Morand C, Crespy V, Demigné C, Texier O, Régérat F, Rémésy C. Quercetin is recovered in human plasma as conjugated derivatives which retain antioxidant properties. FEBS Lett 1998; 426: 331-336

35 Moon J, Tsushida T, Nakahara K, Terao J. Identification of quercetin 3-O$\beta$-D-glucuronide as an antioxidative metabolite in rat plasma after oral administration of quercetin. Free Radic Biol Med 2001; 30: 1274-1285

36 Day AJ, Bao Y, Morgan MR, Williamson G. Conjugation position of quercetin glucuronides and effect on biological activity. Free Radic Biol Med 2000; 29: 1234-1243

37 Shimoi K, Saka N, Nozawa R, Sato M, Amano I, Nakayama T, Kinae N. Deglucuronidation of a flavonoid, luteolin monoglucuronide, during inflammation. Drug Metab Dispos 2001; 29: 1521-1524

38 Cao G, Alessio HM, Cutler RG. Oxygen-radical absorbance capacity assay for antioxidants. Free Radic Biol Med 1993; 14: 303-311

39 Ou B, Hampsch-Woodill M, Prior RL. Development and validation of an improved oxygen radical absorbance capacity assay using fluorescein as the fluorescent probe. J Agric Food Chem 2001; 49: 4619-4626

40 Azorin-Ortuno M, Urban C, Ceron JJ, Tecles F, Gil-Izquierdo A, Pallares FJ, Tomas-Barberan FA, Espin JC. Safety evaluation of an oak-flavored milk powder containing ellagitannins upon oral administration in the rat. J Agric Food Chem 2008; 56: 2857-2865

41 Cerda B, Ceron JJ, Tomas-Barberan FA, Espin JC. Repeated oral administration of high doses of the pomegranate ellagitannin punicalagin to rats for 37 days is not toxic. J Agric Food Chem 2003; 51: 3493-3501

42 Gonzalez-Sarrias A, Azorin-Ortuno M, Yanez-Gascon MJ, Tomas-Barberan FA, Garcia-Conesa MT, Espin JC. Dissimilar in vitro and in vivo effects of ellagic acid and its microbiota-derived metabolites, urolithins, on the cytochrome P450 1A1. J Agric Food Chem 2009; 57: 5623-5632

43 Gonzalez-Sarrias A, Gimenez-Bastida JA, Garcia-Conesa MT, Gomez-Sanchez MB, Garcia-Talavera NV, Gil-Izquierdo A, Sanchez-Alvarez C, Fontana-Compiano LO, Morga-Egea JP, Pastor-Quirante FA, Martinez-Diaz F, Tomas-Barberan FA, Espin JC. Occurrence of urolithins, gut microbiota ellagic acid metabolites and proliferation markers expression response in the human prostate gland upon consumption of walnuts and pomegranate juice. Mol Nutr Food Res 2010; 54: 311-322

44 Seeram NP, Aronson WJ, Zhang Y, Henning SM, Moro A, Lee RP, Sartippour M, Harris DM, Rettig M, Suchard MA, Pantuck AJ, Belldegrun A, Heber D. Pomegranate ellagitannin-derived metabolites inhibit prostate cancer growth and localize to the mouse prostate gland. J Agric Food Chem 2007; 55: 7732-7737

45 Cerda B, Soto C, Albaladejo MD, Martinez P, Sanchez-Gascon F, TomasBarberan F, Espin JC. Pomegranate juice supplementation in chronic obstructive pulmonary disease: a 5-week randomized, double-blind, placebo-controlled trial. Eur J Clin Nutr 2006; 60: 245-253

46 Henning SM, Seeram NP, Zhang Y, Li L, Gao K, Lee RP, Wang DC, Zerlin A, Karp H, Thames G, Kotlerman J, Li Z, Heber D. Strawberry consumption is associated with increased antioxidant capacity in serum. J Med Food 2010; 13: 116-122

47 Seeram NP, Zhang Y, McKeever R, Henning SM, Lee RP, Suchard MA, Li Z, Chen S, Thames G, Zerlin A, Nguyen M, Wang D, Dreher M, Heber D. Pomegranate juice and extracts provide similar levels of plasma and urinary ellagitannin metabolites in human subjects. J Med Food 2008; 11: 390-394

48 Larrosa M, Gonzalez-Sarrias A, Yanez-Gascon MJ, Selma MV, Azorin-Ortuno $M$, Toti S, Tomas-Barberan F, Dolara P, Espin JC. Anti-inflammatory properties of a pomegranate extract and its metabolite urolithin-A in a colitis rat model and the effect of colon inflammation on phenolic metabolism. J Nutr Biochem 2010; 21: 717-725 